\title{
Economic model predictive control without terminal constraints: optimal periodic operation
}

\author{
Matthias A. Müller and Lars Grüne
}

\begin{abstract}
In this paper, we analyze economic model predictive control schemes without terminal constraints, where the optimal operating regime is not steady-state operation, but periodic behavior. We first show by means of two counterexamples, that a classical receding horizon control scheme does not necessarily result in an optimal closed-loop performance. Instead, a multi-step MPC scheme may be needed in order to establish near optimal performance of the closed-loop system. This behavior is analyzed in detail, and we derive checkable dissipativity-like conditions in order to obtain closed-loop performance guarantees.
\end{abstract}

\section{INTRODUCTION}

In recent years, the study of economic model predictive control (MPC) schemes has received a significant amount of attention. In contrast to standard stabilizing MPC, the control objective is the minimization of some general performance criterion, which needs not be related to any specific steadystate to be stabilized. In the literature, closed-loop properties such as performance estimates and convergence issues have been studied for various economic MPC schemes, e.g., in [1, $2,9,12]$ using suitable additional (terminal) constraints, or in [8] without terminal constraints (see also the recent survey article [4]).

A distinctive feature of economic MPC is the fact that the closed-loop trajectories are not necessarily convergent, but can exhibit some more complex, e.g., periodic, behavior. In particular, the optimal operating regime for a given system depends on its dynamics, the considered performance criterion and the constraints which need to be satisfied. The case where steady-state operation is optimal is by now fairly well understood, and various closed-loop guarantees have been established in this case. For example, a certain dissipativity property is both sufficient [2] and (under a mild controllability condition) necessary [13] for a system to be optimally operated at steady-state. The same dissipativity condition (strengthened to strict dissipativity) was used in [1, 2] to prove asymptotic stability of the optimal steady-state for the resulting closed-loop system with the help of suitable terminal constraints. Similar (practical) stability results were established in $[6,8]$ without such terminal constraints.

On the other hand, the picture is still much less complete in case that some non-stationary behavior is the optimal

Matthias A. Müller is with the Institute for Systems Theory and Automatic Control, University of Stuttgart, 70550 Stuttgart, Germany. His work was supported by the German Research Foundation (DFG) within the Cluster of Excellence in Simulation Technology (EXC 310/1) at the University of Stuttgart. matthias.muellerdist.uni-stuttgart.de

Lars Grüne is with the Mathematical Institute, University of Bayreuth, 95440 Bayreuth, Germany. lars. gruene@uni-bayreuth. de operating regime. In [2], it was shown that when using some periodic orbit as (periodic) terminal constraint within the economic MPC problem formulation, then the resulting closed-loop system will have an asymptotic average performance which is at least as good as the average cost of the periodic orbit. Convergence to the optimal periodic orbit was established in $[10,14]$ using similar terminal constraints, and in [11] for linear systems and convex cost functions using less restrictive generalized periodic terminal constraints. Furthermore, dissipativity conditions which are suited as sufficient conditions such that the optimal operating regime of a system is some periodic orbit were recently proposed in [7].

In this paper, we consider economic MPC without terminal constraints for the case where periodic operation is optimal. Using no terminal constraints is in particular desirable in this case as the optimal periodic orbit then needs not be known a priori (i.e., for implementing the economic MPC scheme). Furthermore, the online computational burden might be lower and a larger feasible region is in general obtained. We first show by means of two counterexamples (see Section III), that the classical receding horizon control scheme, consisting of applying the first step of the optimal predicted input sequence to the system at each time, does not necessarily result in an optimal closed-loop performance. We then prove in Section IV that this undesirable behavior can be resolved by possibly using a multi-step MPC scheme instead. In particular, we show that the resulting closed-loop system has an asymptotic average performance which is equal to the average cost of the optimal periodic orbit (up to an error term which vanishes as the prediction horizon increases). This recovers the results of [2], where periodic terminal constraints were used as discussed above. Furthermore, in Section V we derive checkable sufficient conditions in order to apply the results of Section IV. Finally, we close this section by noting that our analysis builds on the one in [8], where closed-loop performance guarantees and convergence results for economic MPC without terminal constraints were established for the case where the optimal operating regime is steady-state operation. However, while some of the employed concepts and ideas are similar to those in [8], various properties of predicted and closed-loop sequences are different in the periodic case considered in this paper, and hence also different analysis methods are required.

\section{PReliminaries And SETUP}

Let $\mathbb{I}_{[a, b]}$ denote the set of integers in the interval $[a, b] \subseteq$ $\mathbb{R}$, and $\mathbb{I}_{\geq a}$ the set of integers greater than or equal to $a$. For 
$a \in \mathbb{R},\lfloor a\rfloor$ is defined as the largest integer smaller than or equal to $a$. The distance of a point $x \in \mathbb{R}^{n}$ to a set $\mathcal{A} \subseteq \mathbb{R}^{n}$ is defined as $|x|_{\mathcal{A}}:=\inf _{a \in \mathcal{A}}|x-a|$. For a set $\mathcal{A} \subseteq \mathbb{R}^{n}$ and $\varepsilon>0$, denote by $B_{\varepsilon}(\mathcal{A}):=\left\{x \in \mathbb{R}^{n}:|x|_{\mathcal{A}} \leq \varepsilon\right\}$. By $\mathcal{L}$ we denote the set of functions $\varphi: \mathbb{R}_{\geq 0} \rightarrow \mathbb{R}_{\geq 0}$ which are continuous, nonincreasing and satisfy $\lim _{k \rightarrow \infty} \varphi(k)=$ 0 . Furthermore, by $\overline{\mathcal{K} L}$ we denote the set of functions $\gamma$ : $\mathbb{R}_{\geq 0} \times \mathbb{R}_{\geq 0} \rightarrow \mathbb{R}_{\geq 0}$ such that for each $\varphi \in \mathcal{L}$, the function $\bar{\gamma}(k):=\gamma(\varphi(k), k)$ satisfies $\bar{\gamma} \in \mathcal{L}$. Note that the definition of a $\overline{\mathcal{K} L}$-function requires weaker properties than those for classical $\mathcal{K} \mathcal{L}$-functions, i.e., each $\mathcal{K} \mathcal{L}$-function is also a $\overline{\mathcal{K} L}$ function (but the converse does not hold).

We consider nonlinear discrete-time systems of the form

$$
x(k+1)=f(x(k), u(k)), \quad x(0)=x
$$

with $k \in \mathbb{I}_{\geq 0}$ and $f: \mathbb{R}^{n} \times \mathbb{R}^{m} \rightarrow \mathbb{R}^{n}$. System (1) is subject to pointwise-in-time state and input constraints $x(k) \in \mathbb{X} \subseteq$ $\mathbb{R}^{n}$ and $u(k) \in \mathbb{U} \subseteq \mathbb{R}^{m}$ for all $k \in \mathbb{I}_{\geq 0}$. For a given control sequence $u=(u(0), \ldots, u(K)) \in \mathbb{U}^{K+1}$ (or $u=$ $\left.(u(0), \ldots) \in \mathbb{U}^{\infty}\right)$, denote by $x_{u}(k, x)$ the corresponding solution of system (1) with initial condition $x_{u}(0, x)=x$. For a given $x \in \mathbb{X}$, the set of all feasible control sequences of length $N$ is denoted by $\mathbb{U}^{N}(x)$, where a feasible control sequence is such that $u(k) \in \mathbb{U}$ for all $k \in \mathbb{I}_{[0, N-1]}$ and $x_{u}(k, x) \in \mathbb{X}$ for all $k \in \mathbb{I}_{[0, N]}$. Similarly, the set of all feasible control sequences of infinite length is denoted by $\mathbb{U}^{\infty}(x)$. In the following, we assume for simplicity that $\mathbb{U}^{\infty}(x) \neq \emptyset$ for all $x \in \mathbb{X}$.

Remark 1: For ease of presentation, we use decoupled state and input constraint sets $\mathbb{X}$ and $\mathbb{U}$ in the statement of our results. Nevertheless, all results in this paper are also valid for possibly coupled state and input constraints, i.e., $(x(k), u(k)) \in \mathbb{Z}$ for all $k \in \mathbb{I}_{\geq 0}$ and some $\mathbb{Z} \subseteq \mathbb{R}^{n} \times \mathbb{R}^{m}$, which will also be used in the examples.

System (1) is equipped with a stage cost function $\ell: \mathbb{X} \times$ $\mathbb{U} \rightarrow \mathbb{R}$, which is assumed to be bounded from below on $\mathbb{X} \times$ $\mathbb{U}$, i.e., $\ell_{\min }:=\inf _{x \in \mathbb{X}, u \in \mathbb{U}} \ell(x, u)$ is finite. Note that this is, e.g., the case if $\mathbb{X} \times \mathbb{U}$ is compact and $\ell$ is continuous. Without loss of generality, in the following we assume that $\ell_{\min } \geq 0$. We then define the following finite horizon averaged cost functional

$$
J_{N}(x, u):=\frac{1}{N} \sum_{k=0}^{N-1} \ell\left(x_{u}(k, x), u(k)\right)
$$

and the corresponding optimal value function

$$
V_{N}(x):=\inf _{u \in \mathbb{U}^{N}(x)} J_{N}(x, u)
$$

In the following, we assume that for each $x \in \mathbb{X}$, a control sequence $u_{N, x}^{*} \in \mathbb{U}^{N}(x)$ exists such that the infimum in (3) is attained, i.e., $u_{N, x}^{*}$ satisfies $V_{N}(x)=J_{N}\left(x, u_{N, x}^{*}\right)$. A standard MPC scheme without additional terminal cost and terminal constraints then consists of minimizing, at each time instant $k \in \mathbb{I}_{\geq 0}$ with current system state $x=x(k)$, the cost functional ${ }^{1}$ (2) with respect to $u \in \mathbb{U}^{N}(x)$ and applying the first part of the resulting optimal input sequence $u_{N, x}^{*}$ to the system. This means that the resulting receding horizon control input to system (1) is given by $u_{M P C}(k):=u_{N, x_{u_{M P C}}(k, x)}^{*}(0)$, where $x_{u_{M P C}}(\cdot, x)$ denotes the corresponding closed-loop state sequence. The finite and infinite horizon averaged cost functionals along this closed-loop state sequence are denoted by $J_{N}^{c l}\left(x, u_{M P C}\right)=$ $\frac{1}{N} \sum_{k=0}^{N-1} \ell\left(x_{u_{M P C}}(k, x), u_{M P C}(k)\right)$ and $J_{\infty}^{c l}\left(x, u_{M P C}\right):=$ $\limsup _{N \rightarrow \infty} J_{N}^{c l}\left(x, u_{M P C}\right)$, respectively.

In [8], it was shown that if system (1) is optimally operated at some steady-state $\left(x^{*}, u^{*}\right)$ with cost $\ell_{0}:=\ell\left(x^{*}, u^{*}\right)$, then under suitable conditions the asymptotic average performance of the closed-loop system, $J_{\infty}^{c l}$, equals $\ell_{0}$ (up to an error term which vanishes as $N \rightarrow \infty$ ). In this paper, we consider the more general case where system (1) is optimally operated at some periodic orbit with period $P \in \mathbb{I}_{\geq 1}$. To this end, consider the following definitions.

Definition 2: A set of state/input pairs $\Pi=$ $\left\{\left(x_{0}^{p}, u_{0}^{p}\right), \ldots,\left(x_{P-1}^{p}, u_{P-1}^{p}\right)\right\}$ with $P \in \mathbb{I}_{\geq 1}$ is called a feasible P-periodic orbit of system (1), if $x_{k}^{p} \in \mathbb{X}$ and $u_{k}^{p} \in \mathbb{U}$ for all $k \in \mathbb{I}_{[0, P-1]}, x_{k+1}^{p}=f\left(x_{k}^{p}, u_{k}^{p}\right)$ for all $k \in \mathbb{I}_{[0, P-2]}$, and $x_{0}^{p}=f\left(x_{P-1}^{p}, u_{P-1}^{p}\right)$. It is called a minimal $P$-periodic orbit if $x_{k_{1}}^{p} \neq x_{k_{2}}^{p}$ for all $k_{1}, k_{2} \in \mathbb{I}_{[0, P-1]}$ with $k_{1} \neq k_{2}$.

In the following, denote by $\Pi_{\mathbb{X}}$ the projection of $\Pi$ on $\mathbb{X}$, i.e., $\Pi_{\mathbb{X}}:=\left\{x_{0}^{p}, \ldots, x_{P-1}^{p}\right\}$.

Definition 3: System (1) is optimally operated at a periodic orbit $\Pi$ if for each $x \in \mathbb{X}$ and each $u \in \mathbb{U}^{\infty}(x)$ the following inequality holds:

$$
\liminf _{T \rightarrow \infty} \frac{\sum_{k=0}^{T-1} \ell\left(x_{u}(k, x), u(k)\right)}{T} \geq \frac{1}{P} \sum_{k=0}^{P-1} \ell\left(x_{k}^{p}, u_{k}^{p}\right)
$$

Definition 3 means that each feasible solution will result in an asymptotic average performance which is as good as or worse than the average performance of the periodic orbit $\Pi$. Furthermore, for $P=1$ the notion of optimal steady-state operation $[2,13]$ is recovered. Note that if system (1) is optimally operated at some periodic orbit $\bar{\Pi}=\left\{\left(\bar{x}_{0}^{p}, \bar{u}_{0}^{p}\right), \ldots,\left(\bar{x}_{P-1}^{p}, \bar{u}_{P-1}^{p}\right)\right\}$, then $\bar{\Pi}$ is necessarily an optimal periodic orbit for system (1), i.e. we have

$$
\sum_{k=0}^{P-1} \ell\left(\bar{x}_{k}^{p}, \bar{u}_{k}^{p}\right)=\inf _{P \in \mathbb{I}_{\geq 1}, \Pi \in S_{\Pi}^{P}} \sum_{k=0}^{P-1} \ell\left(x_{k}^{p}, u_{k}^{p}\right),
$$

where $S_{\Pi}^{P}$ denotes the set of all feasible $P$-periodic orbits.

In case that a system is optimally operated at a periodic orbit $\Pi$, the closed-loop system resulting from application of the economic MPC scheme exhibits optimal performance if $J_{\infty}^{c l}\left(x, u_{M P C}\right)=(1 / P) \sum_{k=0}^{P-1} \ell\left(x_{k}^{p}, u_{k}^{p}\right)$. As discussed in the Introduction, in [2] it was shown that this can be achieved in case that $\Pi_{\mathbb{X}}$ is used as a periodic terminal constraint. When using no terminal constraints, this equality

\footnotetext{
${ }^{1}$ Most MPC schemes in the literature use a non-averaged cost functional, i.e., (2) without the factor $1 / N$. However, since $1 / N$ is just a constant, this does not change the solution of the optimization problem. Here, we use an averaged cost functional for a more convenient statement of our results.
} 
is in general not achieved, as we show in the following by means of some counterexamples. Nevertheless, optimal performance can still be guaranteed also without terminal constraints in case a multi-step MPC scheme is used, as will be shown in Sections IV and V.

\section{MOTIVATING EXAMPLES}

Example 4: Consider the one-dimensional system $x(k+$ $1)=u(k)$ with state and input constraint set $\mathbb{Z}=$ $\{(-1,-1),(-1,0),(0,1),(1,0)\}$ consisting of four elements only and cost $\ell(x, u)$ defined as

$$
\begin{gathered}
\ell(-1,-1)=1, \quad \ell(-1,0)=1, \\
\ell(0,1)=1-2 \varepsilon, \quad \ell(1,0)=1+\varepsilon
\end{gathered}
$$

for some constant $\varepsilon>0$. The system is optimally operated at the two-periodic orbit given by $\Pi=\{(0,1),(1,0)\}$, and with average cost $\ell_{0}:=(1 / 2) \sum_{k=0}^{1} \ell\left(x_{k}^{p}, u_{k}^{p}\right)=1-\varepsilon / 2$. For initial condition $x_{0}=-1$, it follows that for any even prediction horizon $N \in \mathbb{I}_{\geq 2}$, the optimal open-loop input sequence $u_{N, x_{0}}^{*}$ is such $x_{u_{N, x_{0}}^{*}}\left(1, x_{0}\right)=0$ and then $x_{u_{N, x_{0}}^{*}}\left(\cdot, x_{0}\right)$ stays on $\Pi_{\mathbb{X}}$. This means that also the closed-loop system converges to the set $\Pi_{\mathbb{X}}$ and $J_{\infty}^{c l}\left(-1, u_{M P C}\right)=\ell_{0}$. On the other hand, for any odd prediction horizon $N \in \mathbb{I}_{\geq 2}$, the optimal open-loop input sequence $u_{N, x_{0}}^{*}$ is such $x_{u_{N, x_{0}}^{*}}\left(1, x_{0}\right)=-1$, $x_{u_{N, x_{0}}^{*}}\left(2, x_{0}\right)=0$, and then $x_{u_{N, x_{0}}^{*}}\left(\cdot, x_{0}\right)$ stays on $\Pi_{\mathbb{X}}$. But this means that the closed-loop system stays at $x=-1$ for all times, i.e., $x_{u_{M P C}}\left(k, x_{0}\right)=-1$ for all $k \in \mathbb{I}_{\geq 0}$, and hence $J_{\infty}^{c l}\left(-1, u_{M P C}\right)=1>1-\varepsilon / 2=\ell_{0}$.

Example 5: In Example 4, the non-optimal behavior of the closed-loop system for all odd prediction horizons was due to the fact that the cost on the optimal periodic orbit was not constant. The following example shows that the same behavior can occur even if the cost along the optimal periodic orbit is constant. Namely, consider again the system $x(k+1)=u(k)$ with state and input constraint set $\mathbb{Z}=$ $\{(-1,-1),(-1,0),(0,1),(1,0),(1,2),(2,1)\}$ consisting of six elements only and cost $\ell(x, u)$ defined as

$$
\begin{aligned}
& \ell(-1,-1)=1, \quad \ell(-1,0)=1, \quad \ell(0,1)=1-\varepsilon, \\
& \ell(1,0)=1-\varepsilon \quad \ell(1,2)=1-5 \varepsilon, \quad \ell(2,1)=10
\end{aligned}
$$

for some constant $0<\varepsilon<3$. The system is again optimally operated at the two-periodic orbit given by $\Pi=\{(0,1),(1,0)\}$ with average cost $\ell_{0}:=$ $(1 / 2) \sum_{k=0}^{1} \ell\left(x_{k}^{p}, u_{k}^{p}\right)=1-\varepsilon$. Here, starting again at initial condition $x_{0}=-1$, for any even prediction horizon $N \in \mathbb{I}_{\geq 2}$ the optimal open-loop input sequence $u_{N, x_{0}}^{*}$ is such $x_{u_{N, x_{0}}^{*}}\left(1, x_{0}\right)=-1, x_{u_{N, x_{0}}^{*}}\left(2, x_{0}\right)=0$, and then $x_{u_{N, x_{0}}^{*}}\left(\cdot, x_{0}\right)$ stays on $\Pi_{\mathbb{X}}$ until we have $x_{u_{N, x_{0}}^{*}}\left(N, x_{0}\right)=2$. This means that the closed-loop system stays at $x=-1$ for all times, i.e., $x_{u_{M P C}}\left(k, x_{0}\right)=-1$ for all $k \in \mathbb{I}_{\geq 0}$, and hence $J_{\infty}^{c l}\left(-1, u_{M P C}\right)=1>1-\varepsilon=\ell_{0}$. On the other hand, for any odd prediction horizon $N \in \mathbb{I}_{\geq 2}$ similar considerations as above show that the closed-loop system converges to $\Pi_{\mathbb{X}}$, and hence also $J_{\infty}^{c l}\left(-1, u_{M P C}\right)=\ell_{0}$.

The above examples show that the "phase" on the periodic orbit is decisive, i.e., what is the optimal time to converge to the periodic orbit as well as when to leave it again. This results in the fact that one cannot guarantee that for all sufficiently large prediction horizons $N$, the closed-loop asymptotic average performance satisfies $J_{\infty}^{c l}\left(x, u_{M P C}\right)=$ $(1 / P) \sum_{k=0}^{P-1} \ell\left(x_{k}^{p}, u_{k}^{p}\right)$ (plus some error term which vanishes as $N \rightarrow \infty)$, as could be established in [8] for the case of optimal steady-state operation, i.e., $P=1$. On the other hand, one observes in the above examples that if the MPC scheme is modified in such a way that not only the first value of the optimal control sequence is applied to the system, but the first two values, then in both examples the closed-loop system converges to the optimal periodic orbit and hence $J_{\infty}^{c l}\left(x, u_{M P C}\right)=(1 / P) \sum_{k=0}^{P-1} \ell\left(x_{k}^{p}, u_{k}^{p}\right)$, for all prediction horizons $N \in \mathbb{I}_{\geq 2}$. In the following, this will be examined more closely.

\section{Closed-Loop Performance Guarantees}

As mentioned above, in the following we consider a multistep MPC scheme where for some $P \in \mathbb{I}_{\geq 1}$, an optimal input sequence $u_{N, x}^{*}$ is only calculated every $P$ time instants, and then the first $P$ elements of this sequence are applied to system (1). This means that the control input to system (1) at time $k$ is given by

$$
u_{\mathrm{MPC}}(k)=u_{N, x^{\prime}}^{*}([k]),
$$

where $x^{\prime}=x_{u_{\mathrm{MPC}}}(P\lfloor k / P\rfloor, x)$ and $[k]:=k \bmod P$.

Remark 6: The subsequent results are still correct if instead of the $P$-step MPC scheme as defined above, the following variant is used. Namely, an optimal input sequence is computed at each time and only the first element is applied to the system as in standard MPC, but the prediction horizon is periodically time-varying, i.e., $N$ in (2) is replaced by $N-[k]$. By the dynamic programming principle, the closedloop sequences resulting from application of these two schemes are the same. However, the second will in general exhibit better robustness properties in case of uncertainties and disturbances, since feedback is present at each time instant and not only every $P$ time instants.

The first result in this section for the multi-step MPC scheme as defined above is a generalization of Proposition 4.1 in [8].

Proposition 7: Assume there exist $\bar{N}>0$ and $\delta_{1}, \delta_{2} \in \mathcal{L}$ such that for each $x \in \mathbb{X}$ and each $N \geq \bar{N}$ there exists a control sequence $u_{N, x} \in \mathbb{U}^{N+P}(x)$ and time instants $k_{N, x}^{1}, \ldots, k_{N, x}^{P} \in \mathbb{I}_{[0, \ldots, N+P-1]}$ satisfying the following conditions.

(i) The inequality $J_{N}^{\prime}(x) \leq V_{N}(x)+\delta_{1}(N) / N$ holds for

$$
J_{N}^{\prime}(x):=\frac{1}{N} \sum_{\substack{k=0 \\ k \notin\left\{k_{N, x}^{1}, \ldots, k_{N, x}^{P}\right\}}}^{N+P-1} \ell\left(x_{u_{N, x}}(k, x), u_{N, x}(k)\right) .
$$

(ii) There exists $\ell_{0} \in \mathbb{R}$ such that for all $x \in \mathbb{X}$ the following inequality is satisfied:

$$
\frac{1}{P} \sum_{k \in\left\{k_{N, x}^{1}, \ldots, k_{N, x}^{P}\right\}} \ell\left(x_{u_{N, x}}(k, x), u_{N, x}(k)\right) \leq \ell_{0}+\delta_{2}(N)
$$


Then the inequalities

$$
\begin{aligned}
J_{K P}^{c l}\left(x, u_{M P C}\right) & \leq \frac{N}{K P} V_{N}(x)-\frac{N}{K P} V_{N}\left(x_{u_{M P C}}(K P, x)\right) \\
& +\ell_{0}+\delta_{1}(N-P) / P+\delta_{2}(N-P)
\end{aligned}
$$

and

$$
J_{\infty}^{c l}\left(x, u_{M P C}\right) \leq \ell_{0}+\delta_{1}(N-P) / P+\delta_{2}(N-P)
$$

hold for all $x \in \mathbb{X}$, all $N \geq \bar{N}+P$ and all $K \in \mathbb{I}_{\geq 0}$.

Proof: Fix $x \in \mathbb{X}$ and $N \geq \bar{N}+P$. Using the abbreviation $x(k)=x_{u_{M P C}}(k, x)$, from the dynamic programming principle and the definition of the multi-step MPC control input in (5), we obtain that for all $i \in \mathbb{I}_{\geq 0}$

$$
\begin{aligned}
& \sum_{k=0}^{P-1} \ell\left(x(i P+k), u_{M P C}(i P+k)\right) \\
& =N V_{N}(x(i P))-(N-P) V_{N-P}(x((i+1) P)) .
\end{aligned}
$$

Summing up for $i=0, \ldots, K-1$ then yields

$$
\begin{aligned}
& J_{K P}^{c l}\left(x, u_{M P C}\right)=\frac{1}{K P} \sum_{i=0}^{K-1} \sum_{k=0}^{P-1} \ell\left(x(i P+k), u_{M P C}(i P+k)\right) \\
& =\frac{N}{K P} V_{N}(x(0))-\frac{N-P}{K P} V_{N-P}(x(K P)) \\
& +\frac{1}{K P} \sum_{i=1}^{K-1}\left(N V_{N}(x(i P))-(N-P) V_{N-P}(x(i P))\right) .
\end{aligned}
$$

Now consider the summands in (8). Condition (i) of the proposition with $N-P$ in place of $N$ and $x=x(i P)$ implies that $(N-P) V_{N-P}(x(i P)) \geq(N-P) J_{N-P}^{\prime}(x(i P))-$ $\delta_{1}(N-P)$. Furthermore, by optimality of $V_{N}$ we get $V_{N}(x(i P)) \leq J_{N}\left(x(i P), u_{N-P, x(i P)}\right)$. Combining the above and defining $\mathcal{I}:=\left\{k_{N-P, x(i P)}^{1}, \ldots, k_{N-P, x(i P)}^{P}\right\}$, from condition (ii) of the proposition and the definitions of $J_{N}$ and $J_{N}^{\prime}$ we obtain

$$
\begin{aligned}
N & V_{N}(x(i P))-(N-P) V_{N-P}(x(i P)) \\
\leq & N J_{N}\left(x(i P), u_{N-P, x(i P)}\right)-(N-P) J_{N-P}^{\prime}(x(i P)) \\
& +\delta_{1}(N-P) \\
= & \sum_{k \in \mathcal{I}} \ell\left(x_{u_{N-P, x(i P)}}(k, x(i P)), u_{N-P, x(i P)}(k)\right) \\
& +\delta_{1}(N-P) \\
\leq & P \ell_{0}+P \delta_{2}(N-P)+\delta_{1}(N-P) .
\end{aligned}
$$

Recalling that $x(0)=x$ and inserting (9) into (8) for $i=$ $1, \ldots, K-1$ yields $J_{K P}^{c l}\left(x, u_{M P C}\right) \leq N V_{N}(x) /(K P)-$ $(N-P) V_{N-P}(x(K P)) /(K P)+(K-1)\left(\ell_{0}+\delta_{2}(N-P)+\right.$ $\left.(1 / P) \delta_{1}(N-P)\right) / K$. Moreover, using (9) for $i=K$ and dividing by $K P$ yields $-(N-P) V_{N-P}(x(K P)) /(K P) \leq$ $-N V_{N}(x(K P)) /(K P)+\left(\ell_{0}+\delta_{2}(N-P)+(1 / P) \delta_{1}(N-\right.$ $P)) / K$. Together with the above, this results in (6). Finally, (7) follows from (6) by letting $K \rightarrow \infty$ due to the fact that $V_{N}(x(K P)) \geq \ell_{\min }$.

In the following, we construct control sequences $u_{N, x}$ such that Proposition 7 can be applied with $\ell_{0}=$ $(1 / P) \sum_{k=0}^{P-1} \ell\left(x_{k}^{p}, u_{k}^{p}\right)$ for some $P$-periodic orbit $\Pi$. Then, inequality (7) yields the desired property that the asymptotic average performance of the closed-loop system resulting from application of the $P$-step MPC scheme is less than or equal to the average performance of the periodic orbit $\Pi$ (up to an error term which vanishes as $N \rightarrow \infty$ ). As discussed above, this approximately recovers asymptotic average performance results obtained in MPC schemes with (periodic) terminal constraints [2].

Theorem 8: Assume that there exist constants $\ell_{0} \geq 0$, $\bar{\delta}>0$, and $P \in \mathbb{I}_{\geq 0}$ and a set $\mathbb{Y} \subseteq \mathbb{X}$ such that the following properties hold.

(a) There exists $\gamma_{\ell} \in \mathcal{K}_{\infty}$ such that for all $\delta \in$ $(0, \bar{\delta}]$ and all $x \in \mathcal{B}_{\delta}(\mathbb{Y}) \cap \mathbb{X}$ there exists a control sequence $u_{x} \in \mathbb{U}^{P}(x)$ such that the inequality $(1 / P) \sum_{k=0}^{P-1} \ell\left(x_{u_{x}}(k, x), u_{x}(k)\right) \leq \ell_{0}+\gamma_{\ell}(\delta)$ holds.

(b) There exist $N_{0} \in \mathbb{I}_{\geq 0}$ and a function $\gamma_{V} \in \overline{\mathcal{K} \mathcal{L}}$ such that for all $\delta \in(0, \bar{\delta}]$, all $N \in \mathbb{I}_{>N_{0}}$, all $x \in \mathcal{B}_{\delta}(\mathbb{Y}) \cap \mathbb{X}$ and the control sequence $u_{x} \in \mathbb{U}^{P}$ from (a) the inequality $\left|V_{N}(x)-V_{N}\left(x_{u_{x}}(P, x)\right)\right| \leq \gamma_{V}(\delta, N) / N$ holds.

(c) There exist $\sigma \in \mathcal{L}$ and $N_{1} \in \mathbb{I}_{\geq N_{0}}$ with $N_{0}$ from (b) such that for all $x \in \mathbb{X}$ and all $N \in \mathbb{I}_{\geq N_{1}}$, each optimal trajectory $x_{u_{N, x}^{*}}(\cdot, x)$ satisfies $\left|x_{u_{N, x}^{*}}\left(k_{x}, x\right)\right|_{\mathbb{Y}} \leq \sigma(N)$ for some $k_{x} \in \mathbb{I}_{\left[0, N-N_{0}\right]}$.

Then the conditions of Proposition 7 are satisfied.

\section{Proof: See appendix.}

Theorem 8 uses similar conditions as Theorem 4.2 in [8], which were shown to hold in case of optimal steady-state operation. However, there are some crucial differences. Namely, [8, Theorem 4.2] requires that $\left|V_{N}(x)-V_{N}(y)\right| \leq \gamma_{V}(\delta) / N$ has to hold for all $y \in \mathbb{Y}$ and all $x \in \mathcal{B}_{\delta}(\mathbb{Y})$ with $\gamma_{V} \in \mathcal{K}_{\infty}$, which in particular implies that $V_{N}(x)=V_{N}(y)$ for all $x, y \in \mathbb{Y}$, i.e., the optimal value function is constant on $\mathbb{Y}$. In case that $\mathbb{Y}=\Pi_{\mathbb{X}}$ for some periodic orbit $\Pi$, this can in general not be satisfied, as is the case in our motivating examples in Section III. In Theorem 8, condition (b) instead only requires that $\left|V_{N}(x)-V_{N}\left(x_{u_{x}}(P, x)\right)\right| \leq \gamma_{V}(\delta, N) / N$ holds for all $x \in \mathcal{B}_{\delta}(\mathbb{Y}) \cap \mathbb{X}$, where $u_{x}$ is the control sequence from condition (a). Furthermore, $\gamma_{V}$ may depend on $N$, and in particular for fixed $N,\left|V_{N}(x)-V_{N}\left(x_{u_{x}}(P, x)\right)\right|$ needs not go to zero as $\delta \rightarrow 0$, but we only require that $\gamma_{V}(\delta, N) \rightarrow 0$ if both $N \rightarrow \infty$ and $\delta \rightarrow 0$. These relaxations are crucial such that Theorem 8 can be applied with $\mathbb{Y}=\Pi_{\mathbb{X}}$ for some periodic orbit $\Pi$, as shown in the following.

\section{CheCKable SUfFicient CONDITIONS BASED ON DISSIPATIVITY AND CONTROLLABILITY}

It is easy to verify that the two motivating examples satisfy the conditions of Theorem 8 with $\mathbb{Y}=\Pi_{\mathbb{X}}$, which explains the fact that a 2-step MPC scheme results in optimal closedloop performance, as observed in Section III. In general, however, the conditions of Theorem 8 might be difficult to check since they involve properties of optimal trajectories and the optimal value function. The goal of this section is to provide checkable sufficient conditions for conditions (a)-(c) of Theorem 8 for the case where $\mathbb{Y}=\Pi_{\mathbb{X}}$ for some periodic orbit $\Pi$ of system (1). First, we briefly discuss that condition 
(a) follows in a straightforward way from continuity of $f$ and $\ell$. Then, we show that a certain dissipativity-like condition results in a turnpike behavior of the system with respect to the optimal periodic orbit, from which together with suitable controllability assumptions condition (c) follows (see Section V-A). Finally, we discuss in Section V-B how condition (b) can be established under the same dissipativity and controllability assumptions.

Definition 9: System (1) is P-periodic strictly dissipative with respect to a $P$-periodic orbit $\Pi$ if there exist storage functions $\lambda_{0}, \ldots, \lambda_{P-1}: \mathbb{X} \rightarrow \mathbb{R}_{\geq 0}$ and a function $\alpha_{\ell} \in \mathcal{K}_{\infty}$ such that

$$
\begin{aligned}
\lambda_{k+1}(f(x, u))-\lambda_{k}(x) \leq & \ell(x, u)-\ell\left(x_{k}^{p}, u_{k}^{p}\right) \\
& -\alpha_{\ell}\left(|(x, u)|_{\Pi}\right)
\end{aligned}
$$

for all $x \in \mathbb{X}$, all $u \in \mathbb{U}^{1}(x)$, and all $k \in \mathbb{I}_{[0, P-1]}$, with $\lambda_{P} \equiv \lambda_{0}$.

Assumption 10 (Strict dissipativity): System (1) is $P$ periodic strictly dissipative with respect to some $P$-periodic orbit $\Pi$, and the corresponding storage functions $\lambda_{k}, k \in$ $\mathbb{I}_{[0, P-1]}$, are bounded on $\mathbb{X}$.

As was discussed in [7], Assumption 10 is a sufficient condition for system (1) to be optimally operated at the periodic orbit $\Pi$.

Assumption 11 (Local controllability on $B_{\kappa}(\Pi)$ ): There exists $\kappa>0, M^{\prime} \in \mathbb{I}_{\geq 0}$ and $\rho \in \mathcal{K}_{\infty}$ such that for all $z \in \Pi_{\mathbb{X}}$ and all $x, y \in B_{\kappa}(z) \cap \mathbb{X}$ there exists a control sequence $u \in \mathbb{U}^{M^{\prime}}(x)$ such that $x_{u}\left(M^{\prime}, x\right)=y$ and

$$
\left|\left(x_{u}(k, x), u(k)\right)\right|_{\Pi} \leq \rho\left(\max \left\{|x|_{\Pi_{\mathbb{X}}},|y|_{\Pi_{\mathbb{X}}}\right\}\right)
$$

holds for all $k \in \mathbb{I}_{\left[0, M^{\prime}-1\right]}$.

Assumption 12 (Finite time controllability into $B_{\kappa}(\Pi)$ ): For $\kappa>0$ from Assumption 11 there exists $M^{\prime \prime} \in \mathbb{I}_{\geq 0}$ such that for each $x \in \mathbb{X}$ there exists $k \in \mathbb{I}_{\left[0, M^{\prime \prime}\right]}$ and $u \in \mathbb{U}^{k}(x)$ such that $x_{u}(k, x) \in B_{\kappa}(\Pi)$.

Before turning our attention to conditions (b) and (c) of Theorem 8 , we briefly discuss how for the case that $\mathbb{Y}=\Pi_{\mathbb{X}}$ for some $P$-periodic orbit $\Pi \subseteq \operatorname{int}(\mathbb{X} \times \mathbb{U})$, condition (a) with $\ell_{0}=(1 / P) \sum_{k=0}^{P-1} \ell\left(x_{k}^{p}, u_{k}^{p}\right)$ follows from continuity of $f$ and $\ell$. In this case, for each $x \in B_{\delta}(\mathbb{Y})$ for some $\delta \in(0, \bar{\delta}]$, by definition of $\mathbb{Y}$ it holds that $x \in B_{\delta}\left(x_{j}^{p}\right)$ for some $j \in \mathbb{I}_{[0, P-1]}$. Then, if $f$ and $\ell$ are continuous, the control sequence $u_{x} \in \mathbb{U}^{P}$ in condition (a) can be chosen as

$$
u_{x}=\left(u_{j}^{p}, \ldots, u_{P-1}^{p}, u_{0}^{p}, \ldots, u_{j-1}^{p}\right),
$$

and the function $\gamma_{\ell}$ can be computed as follows. As $f$ and $\ell$ are continuous, for each compact set $\mathbb{W} \subseteq \mathbb{X} \times \mathbb{U}$ there exist $\eta_{f}, \eta_{\ell} \in \mathcal{K}_{\infty}$ such that $\left|f(x, u)-f\left(x^{\prime}, u^{\prime}\right)\right| \leq \eta_{f}(\mid(x, u)-$ $\left.\left(x^{\prime}, u^{\prime}\right) \mid\right)$ and $\left|\ell(x, u)-\ell\left(x^{\prime}, u^{\prime}\right)\right| \leq \eta_{\ell}\left(\left|(x, u)-\left(x^{\prime}, u^{\prime}\right)\right|\right)$ for all $(x, u),\left(x^{\prime}, u^{\prime}\right) \in \mathbb{W}$. Choosing $\mathbb{W}$ large enough and $\bar{\delta}>0$ small enough such that $B_{\max \left\{\bar{\delta}, \eta_{f}^{P}(\bar{\delta})\right\}}(\Pi) \subseteq \mathbb{W}$, it is straightforward to show that

$$
\left|x_{u_{x}}(k, x)-x_{[j+k]}^{p}\right| \leq \eta_{f}^{k}(\delta)
$$

for all $k \in \mathbb{I}_{[1, P]}$ and condition (a) of Theorem 8 is satisfied with $\gamma_{\ell}(\delta)=(1 / P) \sum_{k=0}^{P-1} \eta_{\ell}\left(\eta_{f}^{k}(\delta)\right)$.

\section{A. Turnpike behavior with respect to periodic orbits}

We now turn our attention to condition (c) of Theorem 8, which requires that each optimal solution is close to the set $\mathbb{Y}$ for at least one time instant in the interval $\left[0, N-N_{0}\right.$. To this end, we first state the following theorem which establishes a turnpike property [3] for system (1) with respect to a periodic orbit $\Pi$. Turnpike properties with respect to an optimal steady-state have recently been studied in the context of economic MPC both in discrete-time [8] and continuoustime [5]. The following result can be seen as a generalization to the case of time-varying periodic turnpikes.

Theorem 13: Suppose that Assumption 10 is satisfied. Then there exists $C>0$ such that for each $x \in \mathbb{X}$, each $N \in$ $\mathbb{I}_{\geq 1}$, each $\nu>0$, each control sequence $u \in \mathbb{U}^{N}(x)$ satisfying $J_{N}(x, u) \leq(1 / P) \sum_{k=0}^{P-1} \ell\left(x_{k}^{p}, u_{k}^{p}\right)+\nu / N$, and each $\varepsilon>$ 0 the value $Q_{\varepsilon}:=\#\left\{k \in \mathbb{I}_{[0, N-1]}:\left|\left(x_{u}(k, x), u(k)\right)\right|_{\Pi} \leq\right.$ $\varepsilon\}$ satisfies the inequality $Q_{\varepsilon} \geq N-(\nu+C) / \alpha_{\ell}(\varepsilon)$.

Proof: Let $C^{\prime}:=2 \sup _{x \in \mathbb{X}, k \in \mathbb{I}_{[0, P-1]}}\left|\lambda_{k}(x)\right|<\infty, C^{\prime \prime}:=$ $(P-1) \max _{(x, u) \in \Pi} \ell(x, u)$ and $C:=C^{\prime}+C^{\prime \prime}$. In the following, we consider the case $N-(\nu+C) / \alpha_{\ell}(\varepsilon)>0$, as otherwise there is nothing to prove. For a given $N \in \mathbb{I}_{\geq 1}$, define $M$ as the smallest integer such that $M P \geq N$. With this, we obtain

$$
\begin{aligned}
\frac{1}{N} \sum_{k=0}^{N-1} \ell\left(x_{[k]}^{p}, u_{[k]}^{p}\right) & =\frac{M}{N} \sum_{k=0}^{P-1} \ell\left(x_{k}^{p}, u_{k}^{p}\right)-\frac{1}{N} \sum_{k=N}^{M P-1} \ell\left(x_{[k]}^{p}, u_{[k]}^{p}\right) \\
& \geq \frac{1}{P} \sum_{k=0}^{P-1} \ell\left(x_{k}^{p}, u_{k}^{p}\right)-\frac{C^{\prime \prime}}{N}
\end{aligned}
$$

Next, define the rotated cost functions $L_{k}(x, u):=\ell(x, u)-$ $\ell\left(x_{k}^{p}, u_{k}^{p}\right)+\lambda_{k}(x)-\lambda_{k+1}(f(x, u))$ for $k \in \mathbb{I}_{[0, P-1]}$, and note that from the assumption of strict dissipativity, it follows that $L_{k}(x, u) \geq \alpha_{\ell}\left(|(x, u)|_{\Pi}\right)$. Now consider the modified cost functional $\tilde{J}_{N}(x, u):=(1 / N) \sum_{k=0}^{N-1} L_{[k]}\left(x_{u}(k, x), u(k)\right)$. Considering the above, for each control sequence $u \in \mathbb{U}^{N}(x)$ as specified in the theorem we obtain by definition of $L_{k}$ that

$$
\begin{aligned}
\tilde{J}_{N}(x, u)= & J_{N}(x, u)-\frac{1}{N} \sum_{k=0}^{N-1} \ell\left(x_{[k]}^{p}, u_{[k]}^{p}\right) \\
& +(1 / N)\left(\lambda_{0}(x)-\lambda_{[N]}\left(x_{u}(N, x)\right)\right) \\
\leq & J_{N}(x, u)-\frac{1}{P} \sum_{k=0}^{P-1} \ell\left(x_{k}^{p}, u_{k}^{p}\right)+\frac{C^{\prime}+C^{\prime \prime}}{N} \\
\leq & (\nu+C) / N .
\end{aligned}
$$

Now assume for contradiction that $Q_{\varepsilon}<N-(\nu+C) / \alpha_{\ell}(\varepsilon)$. Then there exists a set $\mathcal{N} \subseteq \mathbb{I}_{[0, N-1]}$ of $N-Q_{\varepsilon}>(\nu+$ $C) / \alpha_{\ell}(\varepsilon)$ time instants such that $\left|\left(x_{u}(k, x), u(k)\right)\right|_{\Pi}>\varepsilon$ for all $k \in \mathcal{N}_{\tilde{\tilde{J}}}$ By the assumption of strict dissipativity, this implies that $\tilde{J}_{N}(x, u) \geq\left(N-Q_{\varepsilon}\right) \alpha_{\ell}(\varepsilon) / N>(\nu+C) / N$, which contradicts (14) and hence proves the theorem.

Theorem 13 gives a lower bound $Q_{\varepsilon}$ for the number of time instants where the considered trajectory is "close" to the periodic orbit $\Pi$. This turnpike result can now be used together with the controllability conditions specified 
by Assumptions 11 and 12 to conclude condition (c) of Theorem 8, as shown in the following.

Theorem 14: Suppose that Assumptions 10-12 hold and $\ell$ is bounded on $\mathbb{X} \times \mathbb{U}$. Then condition $(c)$ of Theorem 8 holds for $\mathbb{Y}=\Pi_{\mathbb{X}}$.

Proof: From Assumptions 11 and 12, it follows that for each $x \in \mathbb{X}$ there exists a control sequence $u$ such that the system is steered to a point on $\Pi_{\mathbb{X}}$ in at most $M^{\prime}+M^{\prime \prime}$ steps and then stays on the periodic orbit $\Pi$ for an arbitrary number of time steps. Hence for each $N \in \mathbb{I}_{\geq 1}$ we have for some $j \in \mathbb{I}_{[0, P-1]}$

$$
\begin{aligned}
& V_{N}(x) \leq J_{N}(x, u)=\frac{1}{N}\left(\sum_{k=0}^{N-1} \ell\left(x_{[k+j]}^{p}, u_{[k+j]}^{p}\right)\right. \\
& \min \left\{N, M^{\prime}+M^{\prime \prime}\right\}-1 \\
& \left.+\sum_{k=0} \ell\left(x_{u}(k, x), u(k)\right)-\ell\left(x_{[k+j]}^{p}, u_{[k+j]}^{p}\right)\right) .
\end{aligned}
$$

Using a similar argument as in (13), it follows that $(1 / N) \sum_{k=0}^{N-1} \ell\left(x_{[k+j]}^{p}, u_{[k+j]}^{p}\right) \leq(1 / P) \sum_{k=0}^{P-1} \ell\left(x_{k}^{p}, u_{k}^{p}\right)+$ $C^{\prime \prime} / N$ for all $j \in \mathbb{I}_{[0, P-1]}$. Furthermore, each summand in the second sum of inequality (15) can be upper bounded by $\hat{C}:=\sup _{x \in \mathbb{X}, u \in \mathbb{U}} \ell(x, u)-\min _{(x, u) \in \Pi} \ell(x, u)<\infty$. Hence (15) yields $V_{N}(x) \leq(1 / P) \sum_{k=0}^{P-1} \ell\left(x_{k}^{p}, u_{k}^{p}\right)+\nu / N$ with $\nu:=C^{\prime \prime}+\left(M^{\prime}+M^{\prime \prime}\right) \hat{C}$. Now choose $N_{1}:=N_{0}+1$ and define $\sigma(N)$ arbitrary for $N \in \mathbb{I}_{\left[0, N_{1}-1\right]}$ and $\sigma(N):=$ $\alpha_{\ell}^{-1}\left((\nu+C) /\left(N-N_{0}\right)\right)$ for $N \in \mathbb{I}_{\geq N_{1}}$, with $C$ as defined in the proof of Theorem 13. From the above considerations, it follows that for each $x \in \mathbb{X}$ and each $N \in \mathbb{I}_{\geq N_{1}}$, Theorem 13 can be applied with control sequence $u_{N}^{*}$ and $\varepsilon=\sigma(N)$, resulting in $Q_{\sigma(N)} \geq N-(\nu+C) / \alpha_{\ell}(\sigma(N))=N_{0}$. This means that there are at least $N_{0}$ time instants $k \in \mathbb{I}_{[0, N-1]}$ such that $\left|\left(x_{u_{N}^{*}}(k, x), u_{N}^{*}(k)\right)\right|_{\Pi} \leq \sigma(N)$, and hence also $\left|x_{u_{N}^{*}}(k, x)\right|_{\Pi_{\mathbb{X}}} \leq \sigma(N)$. As there are at least $N_{0}$ such time instants $k$, at least one of these $k$ must satisfy $k \in \mathbb{I}_{\left[0, N-N_{0}\right]}$, i.e., condition (c) of Theorem 8 holds with $k_{x}$ equal to this $k$ and $\mathbb{Y}=\Pi_{\mathbb{X}}$.

Remark 15: Definition 9 is slightly stronger than the usual definition of strict dissipativity. Namely, in Definition 9 "strictness" both with respect to $x$ and $u$ is considered (via the function $\alpha_{\ell}$ in (10)), while typically this is only required with respect to $x$. In fact, the preceding results would still hold in a similar fashion if $\alpha_{\ell}\left(|(x, u)|_{\Pi}\right)$ in (10) was replaced by $\alpha_{\ell}\left(|x|_{\Pi_{\mathbb{X}}}\right)$. In Theorem 13 , the definition of $Q_{\varepsilon}$ would then need to be slightly changed to $Q_{\varepsilon}:=$ $\#\left\{k \in \mathbb{I}_{[0, N-1]}:\left|x_{u}(k, x)\right|_{\Pi_{\mathbb{X}}} \leq \varepsilon\right\}$, which would still be sufficient for establishing Theorem 14. On the other hand, strict dissipativity as in Definition 9 (i.e.,using $\alpha_{\ell}\left(|(x, u)|_{\Pi}\right)$ in (10)) will be needed for the results in Section V-B.

\section{B. Local optimal value function properties}

Next, we turn our attention to condition (b) of Theorem 8 and derive checkable sufficient conditions for it for the case where $\Pi$ is a minimal periodic orbit of system (1). In this case, all state and control sequences satisfying $\left(x_{u}(k, x), u(k)\right) \in \Pi$ for $k \in \mathbb{I}_{[a, b]}$ with $a, b \in \mathbb{I}_{\geq 0}$ must necessarily follow the unique $P$-periodic orbit specified by $\Pi$ during this time interval ${ }^{2}$, i.e., there exists $j \in \mathbb{I}_{[0, P-1]}$ such that $x_{u}(k, x)=x_{[k+j]}^{p}$ and $u(k)=u_{[k+j]}^{p}$ for all $k \in \mathbb{I}_{[a, b]}$. The following auxiliary result shows that also all state and control sequences staying in a sufficiently small neighborhood of $\Pi$ during some time interval must necessarily approximately follow the unique $P$-periodic orbit specified by $\Pi$ during this time interval.

Lemma 16: Let $\Pi$ be a minimal $P$-periodic orbit for system (1), and assume that the function $f$ in (1) is continuous. Then there exists $\bar{\varepsilon}>0$ such that for all $0 \leq \varepsilon<\bar{\varepsilon}$ and each state and control sequence satisfying $\left(x_{u}(k, x), u(k)\right) \in$ $B_{\varepsilon}(\Pi)$ for all $k \in \mathbb{I}_{[a, b]}$ with $a, b \in \mathbb{I}_{\geq 0}$, there exists $j \in$ $\mathbb{I}_{[0, P-1]}$ such that $\left(x_{u}(k, x), u(k)\right) \in B_{\varepsilon}\left(\left(x_{[k+j]}^{p}, u_{[k+j]}^{p}\right)\right)$ for all $k \in \mathbb{I}_{[a, b]}$.

The proof of Lemma 16, which is omitted in this paper due to space restrictions, proceeds by induction to show that if $\left(x_{u}(k, x), u(k)\right) \in B_{\varepsilon}\left(\left(x_{[k+j]}^{p}, u_{[k+j]}^{p}\right)\right)$ for some $j \in \mathbb{I}_{[0, P-1]}$ and some $k \in \mathbb{I}_{[a, b-1]}$, then from continuity of $f$ and the fact that $\min _{x, y \in \Pi_{\mathbb{X}}}|x-y|>0$ it follows that also $\left(x_{u}(k+1, x), u(k+1)\right) \in B_{\varepsilon}\left(\left(x_{[k+1+j]}^{p}, u_{[k+1+j]}^{p}\right)\right)$. With the help of the above, we can now prove the following result.

Theorem 17: Suppose that Assumptions 10 and 11 are satisfied for some minimal $P$-periodic orbit $\Pi \subseteq \operatorname{int}(\mathbb{X} \times$ $\mathbb{U}$ ) of system (1) and with $M^{\prime}=i P$ for some $i \in \mathbb{I}_{\geq 1}$. Furthermore, assume that $f$ and $\ell$ are continuous and that the control sequence $u_{x}$ in condition (a) of Theorem 8 is chosen according to (11). Then condition (b) of Theorem 8 is satisfied for $\mathbb{Y}=\Pi_{\mathbb{X}}$.

Proof: See appendix.

Combining all the above, under the assumptions of strict dissipativity with respect to a periodic orbit $\Pi$, local controllability on a neighborhood of $\Pi$ and finite time controllability into this neighborhood of $\Pi$, it follows that the closed-loop asymptotic average performance is near optimal, i.e., equals the average cost of the periodic orbit $\Pi$ up to an error term which vanishes as $N \rightarrow \infty$. This is summarized in the following corollary.

Corollary 18: Consider the $P$-step MPC scheme as defined via (5) and suppose that Assumptions 10-12 are satisfied for some minimal $P$-periodic orbit $\Pi \subseteq \operatorname{int}(\mathbb{X} \times \mathbb{U})$ of system (1) and with $M^{\prime}=i P$ for some $i \in \mathbb{I}_{\geq 1}$. Furthermore, assume that $f$ and $\ell$ are continuous and $\ell$ is bounded on $\mathbb{X} \times \mathbb{U}$. Then system (1) is optimally operated at the periodic orbit $\Pi$ and there exist $\delta_{1}, \delta_{2} \in \mathcal{L}$ such that the performance estimates (6) and (7) with $\ell_{0}=(1 / P) \sum_{k=0}^{P-1} \ell\left(x_{k}^{p}, u_{k}^{p}\right)$ are satisfied for the resulting closed-loop system.

\section{CONCLUSIONS}

In this paper, we established closed-loop performance bounds for economic MPC without terminal constraints for the case where the optimal operating regime is not stationary, but periodic. While near optimal performance in general cannot be achieved for a classical receding horizon control

\footnotetext{
${ }^{2}$ If $\Pi$ is not minimal, this is not necessarily the case, but different solutions staying inside $\Pi$ for all times might exist.
} 
scheme, this could be established using a $P$-step MPC scheme, with $P$ being the period length of the optimal periodic orbit. This means that the only information about the optimal behavior of the system which is needed a priori (i.e., for implementing the economic MPC scheme) is the period length $P$, but the optimal periodic orbit needs not be known. We conjecture that under the given assumptions, not only performance guarantees can be established as shown in this paper, but also convergence of the resulting closed-loop system to the optimal periodic orbit. This is subject of future research.

\section{APPENDIX}

Proof of Theorem 8: Choose $\bar{N} \in \mathbb{I}_{\geq N_{1}}$ such that $\sigma(\bar{N}) \leq \bar{\delta}$ holds with $\sigma$ from condition (c) of the Theorem. Fix $N \geq \bar{N}$ and consider an arbitrary $x \in \mathbb{X}$ together with the corresponding optimal control sequence $u_{N, x}^{*} \in \mathbb{U}^{N}(x)$ from condition (c). Let $k_{x}$ be the time index from (c), abbreviate $x^{\prime}:=x_{u_{N, x}^{*}}\left(k_{x}, x\right)$ and denote by $u_{x^{\prime}} \in \mathbb{U}^{P}$ the control sequence from condition (a) with $x=x^{\prime}$. Let $x^{\prime \prime}:=x_{u_{x^{\prime}}}\left(P, x^{\prime}\right)$ and let $u_{N-k_{x}, x^{\prime \prime}}^{*}$ be an optimal control sequence for the initial condition $x=x^{\prime \prime}$ and horizon $N-k_{x}$. Using the above, we define the control sequence $u_{N, x} \in \mathbb{U}^{N+P}(x)$ by $u_{N, x}(k):=u_{N, x}^{*}(k)$ for $k \in$ $\mathbb{I}_{\left[0, k_{x}-1\right]}, u_{N, x}(k):=u_{x^{\prime}}\left(k-k_{x}\right)$ for $k \in \mathbb{I}_{\left[k_{x}, k_{x}+P-1\right]}$ and $u_{N, x}(k):=u_{N-k_{x}, x^{\prime \prime}}^{*}\left(k-k_{x}-P\right)$ for $k \in \mathbb{I}_{\left[k_{x}+P, N+P-1\right]}$. This means that $x_{u_{N, x}}(k, x)=x_{u_{N, x}^{*}}(k, x)$ for $k \in \mathbb{I}_{\left[0, k_{x}-1\right]}$ and $x_{u_{N, x}}(k, x)=x_{u_{x^{\prime}}}\left(k-k_{x}, x^{\prime}\right)$ for $k \in \mathbb{I}_{\left[k_{x}, k_{x}+P-1\right]}$. Furthermore, by condition (c) we have $\left|x^{\prime}\right|_{\mathbb{Y}} \leq \sigma(N)$, and from condition (a) it follows that

$$
\begin{aligned}
& \frac{1}{P} \sum_{k=k_{x}}^{k_{x}+P-1} \ell\left(x_{u_{N, x}}(k, x), u_{N, x}(k)\right) \\
& =\frac{1}{P} \sum_{i=0}^{P-1} \ell\left(x_{u_{x^{\prime}}}\left(i, x^{\prime}\right), u_{x^{\prime}}(i)\right) \leq \ell_{0}+\gamma_{\ell}(\sigma(N)) .
\end{aligned}
$$

Moreover, condition (b) of the Theorem implies that for all $K \in \mathbb{I}_{\geq N_{0}}$, we have

$$
V_{K}\left(x^{\prime \prime}\right)=V_{K}\left(x_{u_{x^{\prime}}}\left(P, x^{\prime}\right)\right) \leq V_{K}\left(x^{\prime}\right)+\frac{\gamma_{V}(\sigma(N), N)}{K}
$$

Now distinguish two cases. First, in case that $N-k_{x} \geq 1$, since $N-k_{x} \in \mathbb{I}_{\geq N_{0}}$ by condition (c) we can use (17) with $K=N-k_{x}$ to conclude that

$$
\begin{aligned}
& \frac{1}{N-k_{x}} \sum_{k=k_{x}+P}^{N+P-1} \ell\left(x_{u_{N, x}}(k, x), u_{N, x}(k)\right) \\
& =J_{N-k_{x}}\left(x^{\prime \prime}, u_{N-k_{x}, x^{\prime \prime}}^{*}\right)=V_{N-k_{x}}\left(x^{\prime \prime}\right) \\
& \stackrel{(17)}{\leq} V_{N-k_{x}}\left(x^{\prime}\right)+\frac{\gamma_{V}(\sigma(N), N)}{N-k_{x}} .
\end{aligned}
$$

Setting $k_{N, x}^{i}:=k_{x}+i-1$ in Proposition 7 for $i \in \mathbb{I}_{[1, P]}$, we obtain

$$
\begin{aligned}
J_{N}^{\prime}(x)= & \frac{1}{N} \sum_{k=0}^{k_{x}-1} \ell\left(x_{u_{N, x}^{*}}(k, x), u_{N, x}^{*}(k)\right) \\
& +\frac{1}{N} \sum_{k=k_{x}+P}^{N+P-1} \ell\left(x_{u_{N, x}}(k, x), u_{N, x}(k)\right) \\
\leq & V_{N}(x)-\frac{N-k_{x}}{N} V_{N-k_{x}}\left(x^{\prime}\right) \\
& +\frac{N-k_{x}}{N}\left(V_{N-k_{x}}\left(x^{\prime}\right)+\frac{\gamma_{V}(\sigma(N), N)}{N-k_{x}}\right) \\
= & V_{N}(x)+\gamma_{V}(\sigma(N), N) / N
\end{aligned}
$$

where the above inequality follows from the dynamic programming principle and (18). Hence condition (i) of Proposition 7 is satisfied with $\delta_{1}(N)=\gamma_{V}(\sigma(N), N)$; note that $\delta_{1} \in \mathcal{L}$ as required due to the fact that $\sigma \in \mathcal{L}$ and $\gamma_{V} \in \overline{\mathcal{L N}}$.

Second, if $N-k_{x}=0$, then $J^{\prime}(x)=V_{N}(x)$ and hence condition (i) of Proposition 7 is satisfied for arbitrary $\delta_{1}(N)$.

Finally, by (16) we have that condition (ii) of Proposition 7 is satisfied with $\delta_{2}(N)=\gamma_{\ell}(\sigma(N))$, which concludes the proof of Theorem 8 .

Proof of Theorem 17: As discussed above, for each $x \in B_{\delta}\left(\Pi_{\mathbb{X}}\right)$ for some $\delta \in(0, \bar{\delta}]$, by definition of $\Pi_{\mathbb{X}}$ it holds that $x \in B_{\delta}\left(x_{j}^{p}\right)$ for some $j \in \mathbb{I}_{[0, P-1]}$. Furthermore, (12) yields that $\left|x_{u_{x}}(P, x)-x_{[j+P]}^{p}\right| \leq \eta_{f}^{P}(\delta)$, where $u_{x}$ is the control sequence defined by (11). As $x_{[j+P]}^{p}=x_{j}^{p}$, this implies that both $x$ and $x_{u_{x}}(P, x)$ are contained in the set $B_{\max \left\{\delta, \eta_{f}^{P}(\delta)\right\}}\left(x_{j}^{p}\right)$. Hence a sufficient condition for condition (b) of Theorem 8 to be satisfied is that the inequality $\left|V_{N}(x)-V_{N}\left(x^{\prime}\right)\right| \leq \gamma_{V}(\delta, N) / N$ holds for all $N \in \mathbb{I}_{\geq N_{0}}$, all $y \in \Pi_{\mathbb{X}}$, all $\delta \in(0, \bar{\delta}]$, and all $x, x^{\prime} \in B_{\max \left\{\delta, \eta_{f}^{P}(\delta)\right\}}(y)$. This will be shown in the following.

Choose $\bar{\delta}$ small enough such that $B_{\max \left\{\bar{\delta}, \eta_{f}^{P}(\bar{\delta})\right\}}\left(\Pi_{\mathbb{X}}\right) \subseteq \mathbb{X}$, $\max \left\{\bar{\delta}, \eta_{f}^{P}(\bar{\delta})\right\} \leq \kappa$ and $\rho\left(\max \left\{\bar{\delta}, \eta_{f}^{P}(\bar{\delta})\right\}\right) \leq \bar{\varepsilon}$ with $\kappa$ and $\rho$ from Assumption 11 and $\bar{\varepsilon}$ from Lemma 16. Now consider arbitrary $y \in \Pi_{\mathbb{X}}$ and $x \in B_{\max \left\{\bar{\delta}, \eta_{f}^{P}(\bar{\delta})\right\}}(y)$. For each such $x$, by Assumption 11 there exists a control sequence $u$ such that the system is steered to a point on $\Pi_{\mathbb{X}}$ in $M^{\prime}$ steps and then stays on the periodic orbit $\Pi$ for an arbitrary number of time steps. Using the same argument as in the proof of Theorem 14, this results in the fact that for each $N \in \mathbb{I}_{\geq 1}$, we have $V_{N}(x) \leq J_{N}(x, u) \leq(1 / P) \sum_{k=0}^{P-1} \ell\left(x_{k}^{p}, u_{k}^{p}\right)+a / N$ with $a:=\bar{C}^{\prime \prime}+M^{\prime} \eta_{\ell}\left(\rho\left(\max \left\{\bar{\delta}, \eta_{f}^{P}(\bar{\delta})\right\}\right)\right)$ and $C^{\prime \prime}:=$ $(P-1) \max _{(x, u) \in \Pi} \ell(x, u)$. Now choose $N_{0} \in \mathbb{I}_{\geq 2 M^{\prime}+2}$ large enough such that $\alpha_{\ell}^{-1}\left((a+C)\left(2 M^{\prime}+1\right) /\left(N_{0}-2 M^{\prime}-\right.\right.$ $1)) \leq \min \{\bar{\varepsilon}, \kappa\}$ and such that $\rho\left(\alpha_{\ell}^{-1}\left((a+C)\left(2 M^{\prime}+\right.\right.\right.$ 1) $\left.\left./\left(N_{0}-2 M^{\prime}-1\right)\right)\right) \leq \bar{\varepsilon}$, with $\bar{\varepsilon}$ from Lemma 16 and $\kappa$ and $\rho$ from Assumption 11. In the following, consider an arbitrary $N \in \mathbb{I}_{\geq N_{0}}$. The above inequality for $V_{N}(x)$ implies that we can apply Theorem 13 with $\nu=a$ and $\varepsilon=\alpha_{\ell}^{-1}\left((a+C)\left(2 M^{\prime}+1\right) /\left(N-2 M^{\prime}-1\right)\right)$ to conclude that $\left|\left(x_{u_{N, x}^{*}}(k, x), u_{N, x}^{*}(k)\right)\right|_{\Pi} \leq \varepsilon$ for $Q_{\varepsilon} \geq$ $N-(\nu+C) / \alpha_{\ell}(\varepsilon)=N-N /\left(2 M^{\prime}+1\right)+1=$ $N\left(2 M^{\prime}\right) /\left(2 M^{\prime}+1\right)+1$ time instants $k \in \mathbb{I}_{[0, N-1]}$, and thus 
$\left|\left(x_{u_{N, x}^{*}}(k, x), u_{N, x}^{*}(k)\right)\right|_{\Pi}>\varepsilon$ for at most $\left\lfloor N /\left(2 M^{\prime}+1\right)-1\right\rfloor$ time instants $k \in \mathbb{I}_{[0, N-1]}$. But this implies that there are at least $2 M^{\prime}+1$ consecutive time instants $k \in \mathbb{I}_{[0, N-1]}$ such that $\left|\left(x_{u_{N, x}^{*}}(k, x), u_{N, x}^{*}(k)\right)\right|_{\Pi} \leq \varepsilon$; denote these time instants by $k^{\prime}, \ldots, k^{\prime}+2 M^{\prime}$.

By the above choice of $N_{0}$ and the fact that $N \in \mathbb{I}_{\geq N_{0}}$, Lemma 16 can now be used with $\varepsilon=\alpha_{\ell}^{-1}\left((a+C)\left(2 M^{\prime}+\right.\right.$ $\left.1) /\left(N-2 M^{\prime}-1\right)\right)$ to conclude from the above that there exists $j \in \mathbb{I}_{[0, P-1]}$ such that $\left(x_{u_{N, x}^{*}}(k, x), u_{N, x}^{*}(k)\right) \in$ $B_{\varepsilon}\left(\left(x_{[k+j]}^{p}, u_{[k+j]}^{p}\right)\right)$ for all $k \in \mathbb{I}_{\left[k^{\prime}, k^{\prime}+2 M^{\prime}\right]}$. By continuity of $\ell$ and the fact that $M^{\prime}=i P$ for some $i \in \mathbb{I}_{\geq 1}$, this implies that

$$
\begin{aligned}
& \sum_{k=k^{\prime}}^{k^{\prime}+2 M^{\prime}-1} \ell\left(x_{u_{N, x}^{*}}(k, x), u_{N, x}^{*}(k)\right) \\
& \geq-2 i P \eta_{\ell}(\varepsilon)+2 i \sum_{k=0}^{P-1} \ell\left(x_{k}^{p}, u_{k}^{p}\right) .
\end{aligned}
$$

Furthermore, again due to the fact that $M^{\prime}=i P$ for some $i \in \mathbb{I}_{\geq 1}$, there exists $j \in \mathbb{I}_{[0, P-1]}$ such that $\left(x_{u_{N, x}^{*}}(k, x), u_{N, x}^{*}(k)\right) \in B_{\varepsilon}\left(\left(x_{j}^{p}, u_{j}^{p}\right)\right)$ for $k \in\left\{k^{\prime}, k^{\prime}+\right.$ $\left.M^{\prime}, k^{\prime}+M^{\prime \prime}\right\}$, and hence also $x_{u_{N, x}^{*}}(k, x) \in B_{\varepsilon}\left(x_{j}^{p}\right)$ for $k \in\left\{k^{\prime}, k^{\prime}+M^{\prime}, k^{\prime}+M^{\prime \prime}\right\}$. We can then use Assumption 11 to conclude that there exists a control sequence $u_{2} \in \mathbb{U}^{M^{\prime}}$ such that $x_{u_{2}}\left(M^{\prime}, x_{u_{N, x}^{*}}\left(k^{\prime}, x\right)\right)=x_{u_{N, x}^{*}}\left(k^{\prime}+\right.$ $\left.2 M^{\prime}, x\right)$ and $\left|\left(x_{u_{2}}\left(k, x_{u_{N, x}^{*}}\left(k^{\prime}, x\right)\right), u_{2}(k)\right)\right|_{\Pi} \leq \rho(\varepsilon)$ for all $k \in \mathbb{I}_{\left[0, M^{\prime}-1\right]}$. By choice of $N_{0}$ and the fact that $N \in \mathbb{I}_{\geq N_{0}}$, we have $\rho(\varepsilon) \leq \bar{\varepsilon}$. Hence we can again apply Lemma 16 to conclude that there exists $j \in \mathbb{I}_{[0, P-1]}$ such that $\left(x_{u_{2}}\left(k, x_{u_{N, x}^{*}}\left(k^{\prime}, x\right)\right), u_{2}(k)\right) \in B_{\rho(\varepsilon)}\left(\left(x_{[k+j]}^{p}, u_{[k+j]}^{p}\right)\right)$ for all $k \in \mathbb{I}_{\left[0, M^{\prime}-1\right]}$. Then, using again continuity of $\ell$ and the fact that $M^{\prime}=i P$ for some $i \in \mathbb{I}_{\geq 1}$, it follows that

$$
\begin{aligned}
& \sum_{k=0}^{M^{\prime}-1} \ell\left(x_{u_{2}}\left(k, x_{u_{N, x}^{*}}\left(k^{\prime}, x\right)\right), u_{2}(k)\right) \\
& \leq i P \eta_{\ell}(\rho(\varepsilon))+i \sum_{k=0}^{P-1} \ell\left(x_{k}^{p}, u_{k}^{p}\right) .
\end{aligned}
$$

Now for given $\delta \in(0, \bar{\delta}]$ and $x \in B_{\hat{\delta}}(y)$ with $y \in \Pi_{\mathbb{X}}$ and $\hat{\delta}:=\max \left\{\delta, \eta_{f}^{P}(\delta)\right\}$, consider an arbitrary $x^{\prime} \in B_{\hat{\delta}}(y)$. By Assumption 11, there exists a control sequence $u_{1}$ such that $x_{u_{1}}\left(M^{\prime}, x^{\prime}\right)=x$ and $\left|\left(x_{u_{1}}\left(k, x^{\prime}\right), u_{1}(k)\right)\right|_{\Pi} \leq \rho(\hat{\delta})$ for all $k \in \mathbb{I}_{\left[0, M^{\prime}-1\right]}$. As above, we can use Lemma 16 as well as continuity of $\ell$ and the fact that $M^{\prime}=i P$ for some $i \in \mathbb{I}_{\geq 1}$ to conclude that

$$
\sum_{k=0}^{M^{\prime}-1} \ell\left(x_{u_{1}}\left(k, x^{\prime}\right), u_{1}(k)\right) \leq i P \eta_{\ell}(\rho(\hat{\delta}))+i \sum_{k=0}^{P-1} \ell\left(x_{k}^{p}, u_{k}^{p}\right) .
$$

Combining the above, we now define the following control sequence $\bar{u} \in \mathbb{U}^{N}$ via $\bar{u}(k)=u_{1}(k)$ for $k \in \mathbb{I}_{\left[0, M^{\prime}-1\right]}$, $\bar{u}(k)=u_{N, x}^{*}\left(k-M^{\prime}\right)$ for $k \in \mathbb{I}_{\left[M^{\prime}, k^{\prime}+M^{\prime}-1\right]}, \bar{u}(k)=$ $u_{2}\left(k-k^{\prime}-M^{\prime}\right)$ for $k \in \mathbb{I}_{\left[k^{\prime}+M^{\prime}, k^{\prime}+2 M^{\prime}-1\right]}$, and $\bar{u}(k)=$ $u_{N, x}^{*}(k)$ for $k \in \mathbb{I}_{\left[k^{\prime}+2 M^{\prime}, N-1\right]}$. By construction of $\bar{u}$, we obtain $x_{\bar{u}}\left(k, x^{\prime}\right)=x_{u_{1}}\left(k, x^{\prime}\right)$ for $k \in \mathbb{I}_{\left[0, M^{\prime}-1\right]}$ and
$x_{\bar{u}}\left(M^{\prime}, x^{\prime}\right)=x, x_{\bar{u}}\left(k, x^{\prime}\right)=x_{u_{N, x}^{*}}\left(k-M^{\prime}, x\right)$ for $k \in$ $\mathbb{I}_{\left[M^{\prime}, k^{\prime}+M^{\prime}-1\right]}, x_{\bar{u}}\left(k, x^{\prime}\right)=x_{u_{2}}\left(k-k^{\prime}-M^{\prime}, x_{u_{N, x}^{*}}\left(k^{\prime}, x\right)\right)$ for $k \in \mathbb{I}_{\left[k^{\prime}+M^{\prime}, k^{\prime}+2 M^{\prime}-1\right]}$, and $x_{\bar{u}}\left(k, x^{\prime}\right)=x_{u_{N, x}^{*}}(k, x)$ for $k \in \mathbb{I}_{\left[k^{\prime}+2 M^{\prime}, N-1\right]}$. This yields

$$
\begin{aligned}
& V_{N}\left(x^{\prime}\right) \leq J_{N}\left(x^{\prime}, \bar{u}\right) \stackrel{(21),(22)}{=} \frac{1}{N}\left(i P\left(\eta_{\ell}(\rho(\hat{\delta}))+\eta_{\ell}(\rho(\varepsilon))\right)\right. \\
& +2 i \sum_{k=0}^{P-1} \ell\left(x_{k}^{p}, u_{k}^{p}\right)+\sum_{k=0}^{k^{\prime}-1} \ell\left(x_{u_{N, x}^{*}}(k, x), u_{N, x}^{*}(k)\right) \\
& \left.+\sum_{k=k^{\prime}+2 M^{\prime}}^{N-1} \ell\left(x_{u_{N, x}^{*}}(k, x), u_{N, x}^{*}(k)\right)\right) \\
& \quad \stackrel{(20)}{\leq} V_{N}(x)+\frac{1}{N} i P\left(\eta_{\ell}(\rho(\hat{\delta}))+\eta_{\ell}(\rho(\varepsilon))+2 \eta_{\ell}(\varepsilon)\right) .
\end{aligned}
$$

Defining $\gamma_{V}(\delta, N):=i P\left(\eta_{\ell}(\rho(\hat{\delta}))+\eta_{\ell}(\rho(\varepsilon))+2 \eta_{\ell}(\varepsilon)\right)$ with $\hat{\delta}=\max \left\{\delta, \eta_{f}^{P}(\delta)\right\}$ and $\varepsilon=\alpha_{\ell}^{-1}\left((a+C)\left(2 M^{\prime}+1\right) /(N-\right.$ $\left.2 M^{\prime}-1\right)$ ) results in $V_{N}\left(x^{\prime}\right) \leq V_{N}(x)+\gamma_{V}(\delta, N)$. Exchanging $x$ and $x^{\prime}$ yields the converse inequality $V_{N}\left(x^{\prime}\right) \geq$ $V_{N}(x)-\gamma_{V}(\delta, N)$ and hence $\left|V_{N}\left(x^{\prime}\right)-V_{N}(x)\right| \leq \gamma_{V}(\delta, N)$. Noting that $\gamma_{V}(\delta, N) \in \overline{\mathcal{K} \mathcal{L}}$ as required then concludes the proof of Theorem 17.

\section{REFERENCES}

[1] R. Amrit, J. B. Rawlings, and D. Angeli, "Economic optimization using model predictive control with a terminal cost," Annual Reviews in Control, vol. 35, no. 2, pp. 178-186, 2011.

[2] D. Angeli, R. Amrit, and J. B. Rawlings, "On average performance and stability of economic model predictive control," IEEE Transactions on Automatic Control, vol. 57, no. 7, pp. 1615-1626, 2012.

[3] R. Dorfman, P. A. Samuelson, and R. M. Solow, Linear Programming and Economic Analysis. New York: Dover Publications, 1987, reprint of the 1958 original.

[4] M. Ellis, H. Durand, and P. D. Christofides, "A tutorial review of economic model predictive control methods," Journal of Process Control, vol. 24, no. 8, pp. 1156 - 1178, 2014.

[5] T. Faulwasser, M. Korda, C. N. Jones, and D. Bonvin, "Turnpike and dissipativity properties in dynamic real-time optimization and economic MPC," in Proceedings of the 53rd IEEE Conference on Decision and Control, 2014, pp. 2734-2739.

[6] L. Grüne and M. Stieler, "Asymptotic stability and transient optimality of economic MPC without terminal constraints," Journal of Process Control, vol. 24, no. 8, pp. 1187-1196, 2014.

[7] L. Grüne and M. Zanon, "Periodic optimal control, dissipativity and MPC," in Proceedings of the 21st International Symposium on Mathematical Theory of Networks and Systems, 2014, pp. 1804-1807.

[8] L. Grüne, "Economic receding horizon control without terminal constraints," Automatica, vol. 49, no. 3, pp. 725-734, 2013.

[9] M. Heidarinejad, J. Liu, and P. D. Christofides, "Economic model predictive control of nonlinear process systems using Lyapunov techniques," AIChE Journal, vol. 58, no. 3, pp. 855-870, 2012.

[10] R. Huang, E. Harinath, and L. T. Biegler, "Lyapunov stability of economically oriented NMPC for cyclic processes," Journal of Process Control, vol. 21, no. 4, pp. 501-509, 2011.

[11] D. Limon, M. Pereira, D. Muñoz de la Peña, T. Alamo, and J. M. Grosso, "Single-layer economic model predictive control for periodic operation," Journal of Process Control, vol. 24, no. 8, pp. 1207 1224, 2014.

[12] M. A. Müller, D. Angeli, and F. Allgöwer, "Economic model predictive control with self-tuning terminal cost," European Journal of Control, vol. 19, no. 5, pp. 408-416, 2013.

[13] — "On necessity and robustness of dissipativity in economic model predictive control," IEEE Transactions on Automatic Control, 2015, to appear. DOI: 10.1109/TAC.2014.2361193.

[14] M. Zanon, S. Gros, and M. Diehl, "A Lyapunov function for periodic economic optimizing model predictive control," in Proceedings of the 52nd IEEE Conference on Decision and Control, 2013, pp. 5107 5112. 